\title{
La interpretación dialógica como práctica estratégica. Análisis de la toma de decisiones de cinco intérpretes en los Servicios Públicos
}

\author{
MARTA ARUMÍ RIBAS \\ Universitat Autònoma de Barcelona, Barcelona, España \\ marta.arumi@uab.cat
}

\section{RESUMÉ}

Cet article analyse le comportement stratégique de l'interprète-médiateur chinoisespagnol dans les services publics, concrètement en milieu socioéducatif, relativement à des problèmes de nature diverse (lexiques, culturels, pragmatiques, liés à la gestion de la conversation, éthiques, professionnels). Les données de l'étude ont été obtenues à partir de quinze simulations filmées recréant des situations fréquentes d'interprétation en milieu socioéducatif. Ces données ont été complétées par cinq questionnaires avant tournage et cinq entretiens après tournage réalisés par chacun des participants à l'expérience. L'analyse descriptive a permis d'établir une première catégorie comprenant un certain nombre de ressources stratégiques utilisées par les interprètes. Relativement à une remarquable présence de stratégies de médiation active ainsi qu'à une présence moindre des techniques propres à l'interprétation, l'auteure prône l'établissement d'une distinction précise entre médiation interculturelle et interprétation en milieu social qui assure la transparence et l'autonomie communicative des parties concernées. L'auteure souligne également la pertinence de former les futurs interprètes, de façon consciente, à la prise stratégique de décisions visant à résoudre les problèmes apparus pendant leur performance.

\begin{abstract}
This article examines the strategic behaviour of the Chinese-Spanish public services interpreter-mediator in the socio-educational setting when faced with various types of problems (lexical, cultural, pragmatic, arising from the management of the conversation, and ethical and professional issues). The data were obtained from fifteen filmed simulations which recreated situations that frequently arise in interpreting in the socio-educational field. The data were supplemented by an initial questionnaire and a retrospective interview for the five participants in the study.

The descriptive analysis shows an initial classification of the strategic resources used by the interpreters. Faced with an outstanding use of active mediation strategies and a less frequent use of interpreting techniques, the author argues that it is important to define the difference between intercultural mediation and interpreting in contexts such as this one, to ensure the parties' transparency and autonomy. Moreover, she highlights the importance of consciously training future interpreters on the importance of taking strategic decisions when problem-solving.
\end{abstract}

\section{RESUMEN}

Este artículo analiza el comportamiento estratégico del intérprete-mediador chinoespañol en los servicios públicos del ámbito socioeducativo ante problemas de diversa índole (léxicos, culturales, pragmáticos, derivados de la gestión de la conversación y cuestiones éticas y profesionales). Los datos del estudio se obtuvieron a partir de quince simulaciones filmadas que recreaban situaciones habituales en la interpretación en el ámbito socioeducativo y fueron complementados por cinco cuestionarios pre-tarea y 
cinco entrevistas post-tarea dirigidos a cada uno de los participantes en el experimento. El análisis descriptivo permite establecer una primera categorización de algunos recursos estratégicos que emplean los intérpretes. Frente a una destacada aparición de estrategias de mediación activa y una menor presencia de las técnicas propias de la interpretación, la autora aboga por la importancia de trazar la diferencia entre mediación intercultural e interpretación en contextos como el estudiado para asegurar la transparencia y la autonomía de las partes. Asimismo, destaca la relevancia que tiene formar a los futuros intérpretes de manera consciente sobre la importancia de tomar decisiones estratégicas a la hora de resolver los problemas.

\section{MOTS CLÉS/KEYWORDS/PALABRAS CLAVE}

interprétation dans les services publiques, interprétation dialògiques, stratégies d'interprétation, contexte social et éducatif, interaction

public service interpreting, dialogic interpreting, interpreting strategies, social and educational context, interaction

interpretación en los servicios públicos, interpretación dialógica, estrategias de interpretación, contexto social y educativo, interacción

\section{Introducción}

No cabe duda que la toma de decisiones estratégicas en el proceso interpretativo es esencial y que, en el transcurso de su tarea, el intérprete deberá escoger entre varias estrategias posibles cuando se le presenten los diversos problemas o dificultades que entraña la práctica profesional de la interpretación. Como apunta Kalina, en Pöchchacker (ed. 2015: 402), los procesos estratégicos en comunicación han centrado la investigación en el campo del análisis del discurso monolingüe, la adquisición de la L2 y la traducción, y la investigación existente en estrategias de interpretación deriva de estos enfoques. No obstante, el interés ha solido centrarse en la interpretación simultánea y se ha hecho poca investigación en las estrategias en interpretación dialógica, con la gran excepción de los estudios de Wadensjö (1998). La escasez de estudios descriptivos sobre el uso de estrategias en ISP seguramente se puede achacar a la dificultad que entraña la observación, medida, cuantificación y análisis de los procesos que tienen lugar en cualquier acción estratégica. En este sentido, este artículo pretende ser un paso más, para colmar esta laguna, mediante la descripción de las estrategias de resolución de problemas aplicadas por cinco profesionales ante problemas de interpretación de tipología diversa. Los resultados que presentamos se obtuvieron en el marco de un proyecto de investigación realizado por el grupo de investigación MIRAS ${ }^{1}$ financiado por el Ministerio de Educación y Ciencia de España $^{2}$. Asimismo, el estudio pretende contribuir a los estudios que abordan el contacto de la lengua y cultura chinas en los servicios públicos en España (Sales Salvador 2003; Sánchez Pérez 2009; Sanchez Perez 2015; Vargas-Urpí 2015).

Aunque a partir de ahora nos referiremos a ellos como a intérpretes, en el contexto investigado existe una gran confusión terminológica y una difuminación importante de los roles. Hoy en día conviven en Cataluña y en España varios perfiles profesionales cuyo objetivo es facilitar la comunicación entre las personas inmigradas que desconocen las lenguas oficiales y el personal de los servicios públicos. Bajo lo que podemos denominar el "paraguas de profesionales de la comunicación intercultural mediada" se incluyen traductores e intérpretes, intérpretes en los servicios públicos, mediadores interculturales, mediadores lingüísticos, mediadores interlin- 
güísticos, agentes de acogida, acompañantes lingüísticos, etc. El origen de estas denominaciones y las funciones específicas de cada uno de estos profesionales no están siempre claros. Sin duda, la confusión que rodea a los diferentes perfiles es una característica achacable a la novedad de este tipo de profesiones y a la falta de claridad de los textos legislativos del país que deberían regularlas.

\section{La noción de estrategia en los estudios de Interpretación en los Servicios Públicos}

En la disciplina de la interpretación, la investigación sobre las estrategias se ha centrado tradicionalmente en la modalidad simultánea (Kalina 1998, 2000; Kohn \& Kalina 1996; Sunnari 1995; Riccardi 1996, 1998 y 1999; Gile 2002; Ivanova 2000; Vik-Tuovinen 2002; Gumul 2006; Bartlomiejczyk 2006; Abuín 2007; Arumí 2012; Wang 2012) mientras que el comportamiento estratégico de los intérpretes en los servicios públicos se ha tratado en menor medida y, como explica Kalina (2015), bajo trabajos centrados en la gestión del discurso, los turnos de palabra o la mediación intercultural (Englund Dimitrova 1997; Mason \& Stewart 2001; Roy 2000; Wadensjö 2001). Sin embargo, nadie pone en duda que, en la práctica de la interpretación, cualquiera que sea la modalidad, la toma de decisiones estratégicas es esencial, de ahí la insistencia de la mayoría de los formadores de que el componente estratégico se integre en la formación (Pöchhacker 2010; Li 2003), teniendo presente el papel que juega en la adquisición de la pericia (Moser-Mercer et al. 2000).

Hönig y Kussmaul (1982) definen una estrategia como el proceso que permite encontrar una solución óptima ante un problema de traducción. Hurtado (2001) presenta una definición similar cuando introduce que una estrategia es individual y procedimental y consiste en los mecanismos que utiliza un traductor para solucionar los problemas que se ha encontrado en el proceso de traducción de acuerdo con sus necesidades específicas. En ambos casos, el concepto de estrategia está íntimamente relacionado con el de problema de traducción que, según Nord (1988: 51), son las dificultades objetivas que todos los traductores necesitan resolver durante una tarea, independientemente de su nivel de competencia y de sus condiciones de trabajo formales.

A continuación, repasamos algunos estudios que han tratado la noción de estrategia en ISP, siendo Wadensjö (1998: 107-018) una de las autoras pioneras en tratar de clasificar las prestaciones de los intérpretes en los servicios públicos desde un punto de vista estratégico. Así, establece una distinción entre prestaciones 'cercanas', cercanas al original; prestaciones 'expandidas', que incluyen mayor información que el original; prestaciones 'reducidas', que dan menos información que el original; prestaciones 'sustituidas', que difieren del original; prestaciones 'resumidas', cuando la prestación del intéprete incluye más de una intervención del interlocutor primario; prestaciones multi-parte, cuando la prestación del intérprete se halla dividida entre distintas partes del discurso debido a una interrupción; prestación 'vacía', cuando se trata de una intervención propia del intérprete y prestación 'cero', cuando no se interpreta la intervención del interlocutor primario. Asimismo, Wadensjö (1998: 108110) especifica dos tipos de prestación vacía: “aquella que tiene una orientación textual y que se trata de una intervención propia del intérprete para aclarar dudas o hacer algún comentario sobre la prestación" y "aquella que está enfocada hacia la 
interacción", entre las que incluye las estrategias relacionadas con los turnos de habla, por ejemplo. El componente estratégico es visible en ambas orientaciones porque el intérprete utiliza su propia voz para gestionar los problemas -en la primera para solucionar los problemas relacionados con la comprensión o la expresión y en la segunda (orientación conversacional), en relación con cuestiones de gestión de la interacción.

También Gavioli y Baraldi (2011) estudian las prestaciones vacías, es decir, las intervenciones propias de los intérpretes. Sin embargo, utilizan el término prestaciones 'suspendidas', y se refieren a aquellas interacciones en las que el intérprete empieza una conversación con uno de los interlocutores para clarificar el mensaje que expresará o bien para permitir al interlocutor que amplíe la información que ha dado.

Por su parte, Pöllabauer (2004), en su estudio de las interacciones en el ámbito de las solicitudes de asilo, concluye que todos los intérpretes de la muestra estudiada adoptan las funciones de gestión y coordinación de la conversación, lealtad a los funcionarios, omisión de información que consideran poco relevante, conversaciones monolingües y metacomentarios, estrategias de salvaguarda de la face (reputación de una de las partes), discurso inclusivo, etc. Entre todas estas funciones, Pöllabauer (2004: 163) menciona específicamente las de la salvaguarda de la reputación, a través de las cuales los intérpretes le hacen saber explícitamente a los interlocutores que el mensaje que expresan puede dañar la imagen de una de las partes. En el corpus de datos estudiado por Pöllabauer, esta estrategia se aplica cuando el intérprete, que normalmente utiliza la primera persona, recurre al estilo indirecto y a la tercera persona para expresar esta información.

Merlini y Favaron (2003), en su estudio sobre las estrategias de gestión de poder, se refieren a los cambios en el registro, ya sea cambios hacia el usuario cuando el intérprete muestra empatía hacia él y suele recurrir a simplificar el discurso o bien hacia el proveedor de los servicios, cuando el intérprete adopta un discurso más formal.

Valero-Garcés (2005) se centra específicamente en cuestiones léxicas. En su artículo compara las estrategias que utilizan intérpretes profesionales e intérpretes ad hoc ante problemas de falta de terminología específica en el contexto sanitario. Valero-Garcés (2005: 91) apunta que en ambos casos las estrategias más utilizadas son la omisión, el calco, los préstamos directos, los equivalentes aproximados o provisionales, el recurso a términos no existentes en la lengua de destino, la explicación de los conceptos y el uso de deícticos o de otros recursos extralingüísticos. Estas estrategias léxicas coinciden en gran parte con las que Niska $(1998)^{3}$ había señalado en un estudio anterior.

Por último, los intérpretes entrevistados por Vargas-Urpí (2012) identifican cuatro tipos distintos de problemas: semánticos, pragmáticos, culturales y afectivos. A su vez, la autora añade otros problemas lingüísticos generales como por ejemplo el hecho de que un intérprete no esté familiarizado con la variedad dialectal de una de las partes.

Antes de presentar el estudio, consideramos importante exponer cuál es la aproximación al término estrategia que adoptamos en este artículo. Entendemos las estrategias como aquellas acciones, conscientes o inconscientes, que realizan los intérpretes en la interacción, de manera espontánea, que son válidas en el contexto dialógico en el que tienen lugar y por las que opta el intérprete a la luz de los problemas con los que se encuentra en la comunicación mediada. No diferenciaremos entre 
estrategias (entendidas solo como soluciones positivas) y errores (entendidos, siguiendo a Hurtado 2001, como equivalencias inadecuadas para la tarea encomendada), ya que el objetivo del trabajo no es la evaluación de la calidad sino la descripción de las estrategias empleadas por los intérpretes para hacer frente a los problemas que les puedan surgir en el proceso interpretativo. Asimismo, existe en la bibliografía cierta confusión conceptual con algunas categorías de errores como las omisiones, las adiciones y las estrategias de interpretación y algunos autores insisten en distinguir entre las desviaciones conscientes e inconscientes del original (Napier 2004). En este estudio, tampoco entraremos a valorar la corrección o incorrección de las estrategias empleadas, aunque es evidente que éstas pueden ser acertadas o no y que el empleo de una estrategia no adecuada puede conducir a un error en la interpretación.

\section{El estudio}

\subsection{El experimento}

La investigación, de carácter empírico-experimental, se basó en 15 simulaciones filmadas que recreaban tres situaciones de actuación habituales en el trabajo de los intérpretes en el ámbito socioeducativo en Cataluña. La primera simulación reproducía la entrevista entre una madre china y el tutor de su hijo, escolarizado en una escuela de educación primaria de la provincia de Barcelona. La segunda recreaba una reunión en una oficina de extranjería entre una educadora social, una asistente social y una mujer china abandonada por su marido. Y la tercera, que transcurría en una Oficina Municipal de Escolarización (OME), simulaba la conversación entre una funcionaria de la administración y una mujer china que acababa de llegar a Cataluña y quería escolarizar a sus dos hijos.

Los datos obtenidos de estas 15 simulaciones fueron posteriormente cruzados con los obtenidos a partir de un cuestionario pre-tarea realizado a cada una de las cinco profesionales de la muestra y con una entrevista realizada a cada una de ellas al finalizar la simulación filmada.

La elección de simulaciones en lugar de situaciones reales vino determinada por distintos motivos. Si bien es cierto que las simulaciones presentaban inconvenientes evidentes (artificialidad de la situación, ausencia de imprevistos, menor diversidad de datos observables), tenían la ventaja de constituir una solución creativa al difícil problema del acceso a los datos reales que suele presentar este tipo de interacciones, donde la confidencialidad impide a menudo la presencia de terceras personas en las interacciones y casi siempre la posibilidad de grabarlas. Las simulaciones presentaban asimismo la ventaja de ofrecer un mayor control sobre lo que se quería observar, y permitían la posibilidad de pilotar el guión.

En la primera fase del experimento se analizó una interacción real obtenida de una grabación autorizada que reproducía la interpretación, en un instituto de ESO (Educación Superior Obligatoria), de una entrevista entre una madre china y la tutora de su hijo (Arumí y Vargas 2014). Esta grabación real sirvió de base para elaborar una primera lista de puntos ricos o problemas de interpretación frecuentes en ISP que posteriormente fue corregida y ampliada por los investigadores en el transcurso de varias reuniones de trabajo dedicadas exclusivamente a este tema. Una vez terminada la lista de puntos ricos en ISP, los investigadores diseñaron los guiones correspondientes a cada una de las tres simulaciones previstas, basándose 
en información obtenida tanto de la experiencia de intérpretes profesionales como de la información recabada de otras fuentes.

\subsection{Inclusión de los problemas de interpretación en las simulaciones. La noción de punto rico}

En cada uno de los guiones se introdujo un número determinado de puntos ricos (Beeby et al. 2009), escollos de interpretación que servirían para focalizar, guiar y delimitar el análisis del comportamiento estratégico de los intérpretes. Para realizar la clasificación, se partió de la división entre puntos ricos lingüísticos y puntos ricos extralingüísticos. Así, dentro de lo que sería la concepción lingüística del discurso, hemos considerado los problemas léxicos (ausencia de equivalencia directa, terminología, acrónimos, etc.), los problemas culturales (ausencia de equivalente cultural, detección de malentendidos culturales, etc.) y los problemas pragmáticos (registro, tono, dialecto, ironía, ambigüedad, incoherencia, frases hechas, chistes, bromas, etc.). Por otra parte, dentro del discurso como actividad, hemos considerado los problemas extralingüísticos: los problemas derivados de la gestión de la conversación (gestión de turnos, segmentos demasiado largos, intervención intérprete con voz propia, solapamientos, interrupciones, nuevas incorporaciones a la conversación, silencios, pausas, repeticiones, presentación del intérprete antes de empezar, etc.) y los problemas deontológicos y profesionales (problemas éticos o contrarios a los estándares de calidad de la profesión).

En la siguiente tabla se muestran algunos ejemplos de puntos ricos y su distribución en las tres simulaciones.

TABLA 1

\section{Ejemplo de tabla de Puntos Ricos}

\section{S1-Educación}

S2- OME

S3- Extranjería

\begin{tabular}{|c|c|c|}
\hline Tipo & Ejemplos. ¿Qué hace el intérprete cuando ...? & Simulación \\
\hline \multirow{4}{*}{$\begin{array}{l}\text { Problemas } \\
\text { de tipo } \\
\text { semántico }\end{array}$} & -Una de las partes emplea un elemento lingüístico sin equivalente directo & S2 \\
\hline & $\begin{array}{l}\text {-Una de las partes emplea un término muy especializado (y quizás } \\
\text { desconocido) }\end{array}$ & S1-S2 \\
\hline & $\begin{array}{l}\text {-Una de las partes utiliza un acrónimo o una sigla no relacionados } \\
\text { directamente con el contexto comunicativo, (por ejemplo: "Haremos una } \\
\text { salida al TNC"). }\end{array}$ & S1-S2 \\
\hline & -Una de las intervenciones es ambigua & S1-S2 \\
\hline \multirow{6}{*}{$\begin{array}{l}\text { Problemas } \\
\text { de tipo } \\
\text { pragmático }\end{array}$} & -Una de las partes habla con un registro muy formal o muy especializado & S1-S2 \\
\hline & -Una de las partes habla con un registro muy bajo o muy coloquial & S1 \\
\hline & $\begin{array}{l}\text {-Una de las partes utiliza un tono o estilo irónico o sarcástico. Ej. (si lo } \\
\text { tienes mañana... ¿te puedes dar con un canto en los dientes!) }\end{array}$ & S2 \\
\hline & $\begin{array}{l}\text {-Una de las partes utiliza un gesto facial o corporal con una carga } \\
\text { semántica importante. } \\
\text { Ej. Mirar hacia abajo (el usuario) -¿me está entendiendo?. } \\
\text { Expresión de duda: resoplar. } \\
\text { Gesto de disgusto. } \\
\text { ¿Esto es muy dificil? ¡Uy! }\end{array}$ & S2 \\
\hline & $\begin{array}{l}\text {-La respuesta de una de las partes no es coherente con la pregunta } \\
\text { realizada (posiblemente porque no ha entendido lo que se ha dicho). }\end{array}$ & S3 \\
\hline & -Una de las partes utiliza un dialectalismo. & S3 \\
\hline
\end{tabular}




\begin{tabular}{|l|l|l|}
\hline $\begin{array}{l}\text { Problemas } \\
\text { de tipo } \\
\text { cultural }\end{array}$ & -Una de las partes emplea un elemento cultural sin equivalente directo. & S1 \\
\cline { 2 - 3 } & -Detecta un malentendido de tipo cultural & S2 \\
\hline
\end{tabular}

\subsection{Sujetos participantes}

Se seleccionó a cinco intérpretes del chino al español con experiencia en mediacióninterpretación en el ámbito socioeducativo. Las intérpretes, todas ellas mujeres, tenían un mínimo de 2 años de experiencia en el ámbito de los servicios públicos. Según la información de los cuestionarios previos, dos de ellas habían nacido en Cataluña, una de ellas en Francia (de padres españoles) y dos de ellas en China. Sus lenguas maternas eran el catalán, el español y el chino (respectivamente) y todas las interacciones tuvieron lugar en chino estándar (Putonghua), popularmente conocido como mandarín. Dos de ellas eran graduadas en traducción e interpretación, pero ninguna había recibido formación específica en ISP, aunque tres habían realizado cursos de mediación intercultural.

Una vez seleccionados los sujetos y diseñado el calendario, las simulaciones tuvieron lugar entre febrero y junio de 2014, con una frecuencia aproximada de una grabación al mes. Antes de cada simulación, cada intérprete firmó un consentimiento informado, recibió una serie de instrucciones básicas acerca del experimento y rellenó un cuestionario pre-tarea en el que se recogían datos concretos sobre sus años de experiencia profesional, su formación y sus lenguas de trabajo. A continuación, se les filmó mientras interpretaban del chino al español en las tres simulaciones antes mencionadas, haciendo una pausa de cinco minutos entre cada una. Después de las tres simulaciones, se realizó una entrevista post-tarea en la que se les preguntaba sobre diferentes cuestiones relacionadas con su propia actuación durante el simulacro.

\subsection{Procedimiento de análisis}

A continuación, describimos los pasos que seguimos para tratar los datos y analizarlos.

\subsubsection{Fase 1: Transcripción anotada}

Las simulaciones se transcribieron verbatim en tablas de análisis. Este tipo de transcripción alberga la ventaja de facilitar la comparación entre la intervención original y la interpretación. Las tablas, además, nos permitieron centrar nuestra atención en el análisis de los puntos ricos, detallados explícitamente en una columna.

\subsubsection{Fase 2: Análisis centrado en los puntos ricos}

La segunda fase consistió en:

- describir con detalle lo que ocurre en la prestación en relación con la intervención original.

- examinar específicamente las estrategias empleadas para solucionar los puntos ricos.

- incluir la información procedente de las entrevistas retrospectivas en las que las intérpretes analizaban cómo habían solucionado un problema específico. 


\subsubsection{Fase 3: Análisis de un segundo investigador}

En la tercera fase, un segundo investigador revisó las tablas de análisis, con lo cual se conseguía una triangulación de investigadores (Denzin 1978) y una mayor objetividad en el análisis.

\subsubsection{Fase 4: Transcripción selectiva y análisis de las entrevistas}

Esta cuarta fase consistió en una transcripción selectiva y en el análisis de las entrevistas retrospectivas. Sólo se transcribieron los fragmentos que se consideraron más relevantes, poniendo especial atención en aquellos en los cuales las intérpretes se referían a los puntos ricos. La información de estas entrevistas permitía contextualizar muchas de las decisiones de las intérpretes y aportaban una respuesta objetiva a posibles interrogantes que se planteaban durante el análisis.

\subsubsection{Fase 5: Triangulación y comparación de las simulaciones de distintos intérpretes}

En la fase final del análisis, comparamos las prestaciones de distintos intérpretes en relación al mismo punto rico, con el fin de observar las estrategias que utilizaban para solucionar los problemas. A partir de esta comparación de datos procedentes de fuentes distintas, fue posible realizar un análisis más profundo de las estrategias y presentar una propuesta de clasificación de los distintos tipos de estrategias que detallamos en la siguiente tabla.

TABLA 2

\section{Ejemplo de tabla de triangulación}

\begin{tabular}{|c|c|c|c|c|c|c|}
\hline $\begin{array}{l}\text { Categoría de } \\
\text { punto rico }\end{array}$ & $\begin{array}{l}\text { Punto rico en el } \\
\text { guión }\end{array}$ & Intérprete 1 & Intérprete 2 & Intérprete 3 & Intérprete 4 & Intérprete 5 \\
\hline \multirow[t]{2}{*}{$\begin{array}{l}\text { Discurso } \\
\text { desestructu- } \\
\text { rado }\end{array}$} & $\begin{array}{l}\text { Tutora: } \\
\text { A ver... eh... su } \\
\text { hijo, fuera de las } \\
\text { lenguas, el } \\
\text { castellano y el } \\
\text { catalán, en el } \\
\text { grupo de cuarto } \\
\text { de adaptación, } \\
\text { ¿eh?, que sabemos } \\
\text { que está } \\
\text { adaptado, va bien. } \\
\text { Especialmente en } \\
\text { matemáticas, en } \\
\text { biología, en todas } \\
\text { eh::: todo lo que } \\
\text { son materias más } \\
\text { científicas, no? } \\
\text { Por decirlo de } \\
\text { alguna forma, en } \\
\text { matemáticas }\end{array}$ & $\begin{array}{l}\text { 1. Pregunta } \\
\text { directa para } \\
\text { confirmar } \\
\text { información } \\
\text { Estrategia de } \\
\text { gestión de la } \\
\text { información } \\
\text { 2. Reestructura- } \\
\text { ción del } \\
\text { discurso + } \\
\text { explicaciones } \\
\text { más detalladas } \\
\text { a la usuaria } \\
\text { Estrategia de } \\
\text { gestión de } \\
\text { poder. }\end{array}$ & $\begin{array}{l}\text { Omisiones y } \\
\text { desviación } \\
\text { del original. }\end{array}$ & $\begin{array}{l}\text { Omisiones. } \\
\text { La intérprete } \\
\text { sólo traslada } \\
\text { la informa- } \\
\text { ción esencial. } \\
\text { Toma de } \\
\text { notas. }\end{array}$ & $\begin{array}{l}\text { Omisiones. } \\
\text { Se cambia } \\
\text { "biología" } \\
\text { por "física". } \\
\text { Se pierde la } \\
\text { redundancia } \\
\text { de la } \\
\text { profesora. }\end{array}$ & $\begin{array}{l}\text { 1. Pregunta } \\
\text { directa para } \\
\text { esclarecer } \\
\text { término } \\
\text { (grupo } \\
\text { adaptado) } \\
\text { Estrategia de } \\
\text { gestión de la } \\
\text { información } \\
\text { 2. Reestructu- } \\
\text { ración del } \\
\text { discurso } \\
\text { incluyendo la } \\
\text { información } \\
\text { recibida sobre } \\
\text { el término. }\end{array}$ \\
\hline & $\begin{array}{l}\text { Información } \\
\text { procedente de la } \\
\text { entrevista } \\
\text { retrospectiva }\end{array}$ & & $\begin{array}{l}\text { "En la vida } \\
\text { real habría } \\
\text { explicado } \\
\text { más cosas." }\end{array}$ & $\begin{array}{l}\text { No lo percibe } \\
\text { como } \\
\text { dificultad. }\end{array}$ & $\begin{array}{l}\text { Intenta } \\
\text { extraer la } \\
\text { idea general. }\end{array}$ & \\
\hline
\end{tabular}




\begin{tabular}{|c|c|c|c|c|c|c|}
\hline \multirow{2}{*}{$\begin{array}{l}\text { Culturema: } \\
\text { en China, las } \\
\text { notas se } \\
\text { calculan } \\
\text { sobre } 100 \text { y } \\
\text { para aprobar } \\
\text { es necesario } \\
\text { un } 60 \text {, } \\
\text { mientras que } \\
\text { en España el } \\
\text { baremo es de } \\
10 \text { y para } \\
\text { aprobar se } \\
\text { necesita un } \\
5 .\end{array}$} & $\begin{array}{l}\text { Tutora: } \\
\text { Las tiene } \\
\text { suspendidas con } \\
\text { un cuatro, tres y } \\
\text { medio-cuatro }\end{array}$ & $\begin{array}{l}\text { Adición (la } \\
\text { intérprete } \\
\text { explica que la } \\
\text { nota "no está } \\
\text { muy mal") para } \\
\text { contextualizar } \\
\text { que se trata de } \\
\text { un aprobado } \\
\text { alto. } \\
\text { Estrategia de } \\
\text { gestión de } \\
\text { poder. }\end{array}$ & $\begin{array}{l}\text { No se } \\
\text { explica. }\end{array}$ & $\begin{array}{l}\text { Se añade } \\
\text { información } \\
\text { contextual: } \\
\text { "con cinco ya } \\
\text { es aprobado". } \\
\text { + Toma de } \\
\text { notas. } \\
\text { Estrategia de } \\
\text { gestión de } \\
\text { poder + } \\
\text { estrategia de } \\
\text { memoria }\end{array}$ & $\begin{array}{l}\text { Se simplifica } \\
\text { (tres-cuatro) } \\
\text { y no se añade } \\
\text { ninguna } \\
\text { explicación. }\end{array}$ & $\begin{array}{l}\text { Se añade } \\
\text { explicación } \\
\text { sobre la nota } \\
\text { necesaria } \\
\text { para aprobar. } \\
\text { Estrategia de } \\
\text { gestión de } \\
\text { poder }\end{array}$ \\
\hline & $\begin{array}{l}\text { Información } \\
\text { procedente de la } \\
\text { entrevista } \\
\text { retrospectiva }\end{array}$ & $\begin{array}{l}\text { Normalmente } \\
\text { lo explica más y } \\
\text { busca } \\
\text { equivalentes en } \\
\text { el sistema chino } \\
\text { (Empowerment) }\end{array}$ & & & & $\begin{array}{l}\text { Contextuali } \\
\text { zación } \\
\text { - gestión de } \\
\text { poder. }\end{array}$ \\
\hline \multirow[t]{2}{*}{ Teminología } & $\begin{array}{l}\text { "Graduado de } \\
\text { ESO» }\end{array}$ & $\begin{array}{l}\text { 中学毕业证书 } \\
\text { Certificado de } \\
\text { graduado de } \\
\text { secundaria } \\
\text { Equivalente } \\
\text { (más general } \\
\text { que el original) }\end{array}$ & Omisión & $\begin{array}{l}\text { 学校证书, 毕 } \\
\text { 业证 } \\
\text { Certificado } \\
\text { escolar, } \\
\text { título de } \\
\text { graduado } \\
\text { Equivalentes } \\
\text { (más } \\
\text { generales } \\
\text { que el } \\
\text { original) }\end{array}$ & $\begin{array}{l}\text { 毕业证书 } \\
\text { Certificado } \\
\text { de gradua- } \\
\text { ción, aunque } \\
\text { en la frase } \\
\text { anterior } \\
\text { indica “de } \\
\text { cuarto de } \\
\text { primaria". } \\
\text { Hiperónimo }\end{array}$ & $\begin{array}{l}\text { 初中毕业证书 } \\
\text { Certificado de } \\
\text { graduación } \\
\text { del primer } \\
\text { ciclo de } \\
\text { secundaria. } \\
\text { Equivalente } \\
\text { (es el más } \\
\text { preciso) }\end{array}$ \\
\hline & $\begin{array}{l}\text { Información } \\
\text { procedente de la } \\
\text { entrevista } \\
\text { retrospectiva }\end{array}$ & $\begin{array}{l}\text { Este es el } \\
\text { término que se } \\
\text { utiliza } \\
\text { habitualmente. }\end{array}$ & & & & \\
\hline
\end{tabular}

\section{Resultados}

En este apartado presentamos los principales resultados de la investigación, divididos en las dos grandes categorías que han guiado el estudio. Por un lado, los puntos ricos lingüísticos y, por el otro, los puntos ricos extralingüísticos, que recogen aquellos puntos ricos propios del discurso dinámico, que se construye entre dos o más interlocutores que formulan preguntas, se interrumpen, solapan sus discursos, etc. Analizamos cuáles son las estrategias que han aplicado las intérpretes para cada uno de los puntos ricos. Para realizar la clasificación de los datos y categorización en estrategias, hemos seguido la propuesta de Arumí (2012) así como las de Wadensjö (1998), Gavioli y Baraldi (2011) y Valero-Garcés (2005), basada en Niska (2002).

\subsection{Puntos ricos lingüísticos}

\subsubsection{Puntos ricos léxicos}

El primer grupo de problemas considerados, y también el más numeroso, es el que está relacionado con los problemas terminológicos, es decir, cuando las intérpretes se hallan ante términos especializados propios del ámbito en el que están trabajando. En concreto, entre las tres simulaciones, se introdujeron 20 puntos ricos léxicos, que dieron lugar a un total de 116 estrategias detectadas. Cabe notar que para un mismo punto rico puede ser que una intérprete aplique más de una estrategia de resolución, con lo cual se han considerado tantas estrategias como se hayan aplicado. 
GRÁFICO 1

Estrategias de resolución de problemas terminológicos



La principal estrategia usada por las intérpretes es la omisión (en 33 ocasiones) seguida de la neutralización (21), la traducción perifrástica (20) y el recurso a equivalentes funcionales (18). En el caso de la omisión, es difícil llegar a establecer si ésta es consciente o inconsciente, puede ser que la intérprete haya olvidado el término, que no lo haya comprendido o simplemente puede ser una estrategia para evitar un término que no le es familiar. Solamente en un caso, en la entrevista retrospectiva, la intérprete afirmaba que había omitido el término "pensión alimenticia" porque no lo conocía en chino y porque aparecía en una idea con mucha información con lo cual había decidido dar prioridad a otros elementos de esa intervención.

En las ocasiones en las que encontramos neutralización, siempre existe una pérdida de precisión al decidir generalizar. Asimismo, en dos ocasiones, una de las estrategias que las intérpretes utilizan para neutralizar es el recurso a los deícticos (este, aquí) que les sirven para solucionar la aparición de un término que desconocen, estrategia que también había detectado Valero-Garcés en su estudio de 2005.

Vale la pena destacar el uso extendido de la traducción perifrástica, que, por otra parte, tiene sentido en un contexto en el que es difícil acceder de forma inmediata a materiales de referencia que puedan facilitar una traducción más precisa. En algunos casos, algunas intérpretes preguntaron al proveedor el significado de un término y la traducción perifrástica se basa en la definición del término que reciben (por ejemplo, la intérprete 3 preguntó por la definición de "ciclo formativo de grado medio"). En la entrevista retrospectiva, esta intérprete explica que básicamente ha trabajdo en el contexto social y que, por esta razón, la mayoría de términos específicos del ámbito educativo no le son familiares, con lo cual recurre a hacer muchas preguntas al proveedor.

Cuando se trata de la aparición de nombres propios (calle Pez, Sant Quirze, Ripollet, etc.), las estrategias utilizadas por las intérpretes son de carácter bastante distinto. Concretamente, se dan 4 puntos ricos en los que aparecen nombres propios, con un total de 20 situaciones en las que detectamos una estrategia de resolución. A diferencia de las cuestiones terminológicas, al tratarse de nombres propios, las intérpretes tienen una mayor tendencia a la formulación de preguntas directas concretas, para mejorar la comprensión del nombre propio (en 7 ocasiones). Asimismo, también recurren a la neutralización (4), a la omisión (4) y al equivalente funcional (4). 
GRÁFICO 2

Estrategias de resolución de problemas con los nombres propios

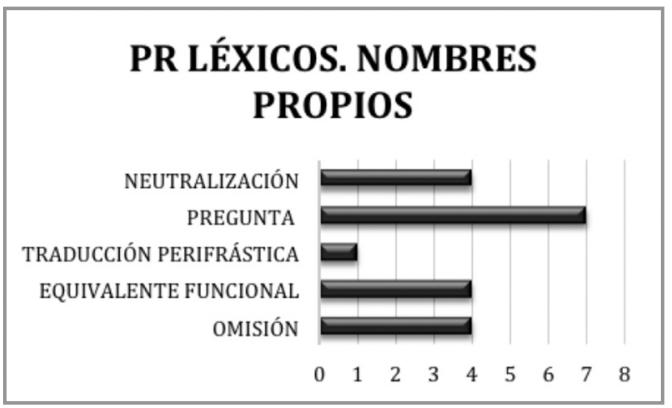

Por último, en relación al uso de siglas (CEIP, PIRMI), que se da en 2 puntos ricos -lo cual supone 10 intervenciones estratégicas-, a diferencia de lo que sería de esperar, la estrategia lógica aplicada por las intépretes no es la de recurrir a una pregunta, que permita una repetición de la sigla, sino a la neutralización en primer lugar (en 4 ocasiones) y al uso del término en la lengua original (en 3 ocasiones). En relación a la neutralización, observamos como, por ejemplo, cuando en el discurso original se habla de CEIP (Centro de Educación Infantil y Primaria) las intérpretes optan por el hiperónimo "escuela" o bien observamos como el PIRMI (Renta Mínima de Inserción) se convierte en un término más general: subsidio o ayuda.

\subsubsection{Puntos ricos culturales}

La tendencia generalizada de las intérpretes es a explicar, a través de una prestación expandida (Wadensjö 1998), los elementos culturales, la mayoría de las veces sin que una de las partes sea consciente de que se está añadiendo esta explicación. Concretamente, de las 15 intervenciones ( 3 puntos ricos culturales) en las que se plantean estrategias de resolución de conflictos culturales, en siete de ellas la intérprete añade información y una explicación cultural.

En el siguiente extracto, procedente de la simulación que recrea la entrevista entre una tutora y una madre china, recogemos el momento en el que la tutora comunica a la madre que su hijo ha suspendido las asignaturas de lengua castellana y lengua catalana pero, sin embargo, le transmite que las llegará a aprobar.

Extract 1. T=tutora; $\mathrm{I} 1=$ intérprete $1 ; \mathrm{I} 2=$ =intérprete 2

T: A ver ... ahora, eh ... las que tiene suspendidas son con cuatros, ¿eh?, que son el castellano y el catalán, básicamente, son tres y medio -cuatro. O sea que llegará a aprobar.

I1: 没及格的课程就是有两个都是四分多, 四分多就是西班牙语根加泰兰与是没及格, 但是不是很差, 还可以, 老师, 班主任, 老师说他可以毕业, 可以及格这两个课 程。

A ver (.) él las que no ha aprobado son español y catalán, pero estas notas no son muy bajas, son un tres y medio - cuatro, con cinco ya es nota, ya es aprobado, ya es superado.

I2: 她说现在呢他还没有过那些课程就是西班牙语跟加泰罗尼亚语, 还是语言那方面 嘛, 就是他的分就是三点五四分, 因为在西班牙满分不是一百分, 在这里是十分嘛, 他 得到是三点五、四::: 
Ella dice ahora que las que no ha aprobado son el español y el catalán, las asignaturas de lenguas, que tiene un tres y medio - cuatro; porque en España la nota máxima no es cien, sino que aquí es diez, y él tiene un tres y medio - cuatro

Recogemos la actuación de dos profesionales (I1 e I2). En el caso de la intérprete 1, si bien no añade directamente una explicación cultural, sí que, a partir del conocimiento que tiene de ambas realidades, añade un juicio de valor "pero estas notas no son muy bajas", con lo que además persigue tranquilizar a la madre. I2 opta por añadir una explicación cultural referida al sistema de calificación "en España la nota máxima no es cien, sino que aquí es diez". La estrategia de la prestación expandida tiene en ambos casos el objetivo de contextualizar la referencia cultural. En las entrevistas a posteriori, ambas profesionales hacen referencia a que el sistema chino de calificación es muy distinto al español y que sienten que deben incluir una ayuda cultural que permita a las madres situarse en el nuevo sistema educativo.

No obstante, hay que destacar que, en la mayoría de los casos, una vez se ha añadido la información, no existe una transparencia de información hacia los dos interlocutores, de tal modo que se haga notar al otro interlocutor en cuestión que se está añadiendo información para superar una brecha cultural.

Por otro lado, es preocupante que también en 7 ocasiones las intérpretes no detecten los retos culturales que se les plantean, con lo cual la información no llega a su destinatario (normalmente el proveedor que desconoce la cultura china).

\subsubsection{Puntos ricos pragmáticos}

En relación a la pragmática, consideramos en primer lugar aquellos momentos en que el intérprete se encuentra un discurso de partida desestructurado. Concretamente, se incluyeron dos puntos ricos de este tipo, que dan lugar a 13 movimientos estratégicos. Es muy importante señalar que solo las intérpretes que han preguntado por la repetición de la información (en dos ocasiones) para mejorar la comprensión, han sido capaces de aportar la información completa y reestructurada. Asimismo, en cuatro ocasiones las intérpretes han recurrido a la toma de notas. Cuando no hay aplicación de la toma de notas, las intérpretes recurren al resumen porque no son capaces de transmitir de memoria el mensaje completo. En el ejemplo siguiente, observamos como, a pesar de que el discurso de la tutora resulte bastante desestructurado, la intérprete consigue transformarlo en un discurso estructurado en el que detecta cuál es la información principal que la tutora ha querido transmitir:

\section{Extracto 2. T=tutora; I=intérprete}

T: A ver... eh... su hijo, al margen de las lenguas, el castellano y el catalán, en el grupo de cuarto de adaptación, ¿eh?,, que sabemos que está adaptado, va bien. Especialmente en matemáticas, en biología, en todas eh ... todo lo que son materias más científicas, no? Para decirlo de alguna manera, en matemáticas.

$\mathrm{I}$ : 就是你的儿子初四, 现在上初四就是水平是跟其他的水平是适应他的水平, 不是跟 本级人一样的水平啊, 那学习的课程除了语言以外, 语言西班牙语, 加泰兰与还不怎么 样其他的呢, 科学方面的课程就不错, 特别是数学啊, 数学很不错。

A ver, tu hijo, que está en cuarto de secundaria, su nivel en comparación con otros, su nivel es adaptado, no es el mismo que el de las personas de su mismo nivel. Entonces, en las asignaturas que estudia, a parte de las lenguas, la lenguas, el castellano y el catalán 
no le van muy bien, en las otras, las asignaturas de ámbito científico no le van mal, sobre todo las matemáticas, las matemáticas no le van nada mal.

Por otro lado, también se introdujeron tres puntos ricos relacionados con contenidos incoherentes. De los 21 movimientos estratégicos que detectamos, diez de ellos consisten en que las intérpretes formulan una pregunta directa que les permite mejorar la comprensión y asegurarse de lo que han entendido. De la misma manera, es frecuente recurrir a una triple estrategia que consiste en formular una pregunta que permite corroborar la comprensión, resumir y omitir la información que se sigue valorando como inconnexa. Lo vemos en el ejemplo siguiente en el que a la intérprete no le ha quedado claro si la madre se está refiriendo a si es el hijo quien desea cursar bachillerato o bien si es el padre que quiere que su hijo lo haga. En este sentido, la mediadora formula una pregunta directa a la madre y, una vez la madre se lo aclara, opta por una intervención en la que resume la información, aportando solo la información básica y dejando de lado la información que estima incoherente:

Extract 3. $M=$ madre; I=intérprete

M: 因为我也讲过, 我说到高中以后就是选择大学, 大学里面你会说, 你不用说, 他大 学都会进的, 然后也许努力在高专里面, 他说他还是选高中, 高中是直接读大学。

Porque yo también lo decía, decía que después del bachillerato se elige la universidad. Y en la universidad vas a decirme que, no hace falta que me lo comentes, que puede entrar en la universidad, después si se aplica en el ciclo formativo superior, él dice que sigue queriendo hacer el bachillerato, que con el bachillerato se va directamente a la universidad.

I: (a la madre) 你的儿子说是吧? 他要上高中, 是你儿子说的还是你丈夫?

Lo dice tu hijo, ¿verdad? Que quiere hacer bachillerato, ¿lo dice tu hijo o tu marido?

I: Mhm. El hijo también lo dice, ¿eh? Él también dice que ... Que le gustaría hacer bachillerato.

$\mathrm{M}$ : 因为我也说过, 读高中是直接读大学。

Yo también se lo he dicho, que si haces bachillerato entras directo a la universidad.

I: 你儿子呢? 怎么说? 他也:::?

¿Y tú hijo? ¿Qué dice? ¿Él también...

$\mathrm{M}$ : 他说他还是选高中。

I: Él dice que prefiere escoger bachillerato

I: ¡Vale! Que los tres, ¿eh?, que los tres de la familia, la madre, sí, también, vale, de acuerdo con la formación profesional, con el grado media, pero que mejor (.) claro, mejor hacer bachillerato, mejor porque es la manera más rápida, no? y además (.) directa para ir a la universidad.

A su vez, dos de las intérpretes deciden transmitir la información incoherente, sin ningún tipo de pregunta ni consulta previa.

Cuando los puntos ricos consisten en introducir intervenciones largas (5 puntos ricos y un total de 25 intervenciones estratégicas), la principal solución a la que recurren las intérpretes es a la omisión (en 21 ocasiones). En este caso, las omisiones son debidas, en primer lugar, al poco recurso de las profesionales a la toma de notas (solo 
en 4 ocasiones) y, en segundo lugar, a la poca tendencia que tienen a interrumpir al proveedor o al usuario. Además, merece la pena destacar también la estrategia del resumen, puesto que, en intervenciones largas, las intérpretes suelen decidir prescindir de una parte del mensaje y concentrarse en aquellas partes que saben que son las que provocarán la reacción del otro interlocutor. En terminología de Davidson (2000), las intérpretes estarían actuando de gatekeeper, con el objetivo de seleccionar sólo aquella información que, a su entender, está más relacionada con el tema que les ocupa. En las entrevistas retrospectivas, estas afirman haberlo aplicado como estrategias conscientes, para no abrumar a la usuaria con tanta información y no confundirla.

Por último, en el punto rico que consistió en dejar una idea sin terminar, observamos como dos de las intérpretes deciden completar la idea añadiendo información contextual y, en cambio, tres de las profesionales deciden omitir toda la idea.

\subsection{Puntos ricos extralingüísticos}

\subsubsection{Puntos ricos en la gestión de la conversación}

En lo que respecta a la gestión de la conversación, hemos tenido en cuenta las interrupciones y los solapamientos. Las interrupciones se introdujeron en dos puntos ricos que dan lugar a 10 movimientos estratégicos. Llama la atención el hecho de que en ocho de estas reacciones estratégicas la opción de las intérpretes sea retomar la información, a pesar de la interrupción que se ha producido, normalmente ayudándose del apoyo de la toma de notas. En dos ocasiones se produce una omisión de la información, que no se retoma tras la interrupción. En el siguiente extracto, vemos que, mientras la intérprete está formulando el mensaje en chino, la tutora la interrumpe y añade la información "un título de técnico medio, técnico de grado medio, ¿eh?”, con lo cual la intérprete deja de interpretar, formula una pregunta directa a la tutora para corroborar la información que ésta ha añadido (perdona, igrado de...?) y a continuación retomará esta información en su siguiente intervención.

\section{Extracto 4. T=tutora; $\mathrm{I}=$ intérprete}

$\mathrm{T}$ : $\mathrm{Y}$ en dos años ... dos son dos mil horas, normalmente son casi dos años, ya tiene una titulación profesional, como mínimo, para poder buscar un trabajo con un cierto ya con un cierto ... bueno, dijéramos nivel, no? No quiere decir que sea mucho pero... ya no es nada, no?

\section{I: 学习过程应该一共两年一共一千个小时}

El proceso de estudio es de unos dos años, en total son mil horas

T: Un titulo de técnico medio, técnico de grado medio, ¿eh?

I: (a la madre) 上这两年的培训学习啊以后可以获得技术 (.) 不好意思。

Después de estos dos años de estudio y formación, podrá obtener un título de...

I: (a la tutora) Perdona, ¿grado de...?

T: Técnico de grado medio.

I: (a la madre) 中等水平的科技人员学凭嘛 (.) 算是一个学凭证书吧

Título de personal científico-técnico de grado medio (.) como un certificado con el título 
En cuanto a los solapamientos, se introdujeron en un punto rico. En todos los casos (5 reacciones estratégicas) el resultado fue el mismo: una omisión directa de la primera parte del contenido del solapamiento.

\subsubsection{Puntos ricos deontológicos}

Cinco de los puntos ricos giraban alrededor de un conflicto deontológico y de los 25 turnos en los que identificamos que se podían haber tenido en cuenta principios éticos, solo en 3 ocasiones las intérpretes respetan dichos códigos. Las estrategias detectadas ante problemas de tipo ético o deontológico están vinculadas a tres cuestiones específicas: la delimitación del rol del intérprete (hasta dónde puede o debe actuar, cuáles son sus fronteras profesionales), el comportamiento ante determinados comentarios discriminatorios o racistas y la transparencia.

En dos ocasiones, se proponía a la intérprete ir más allá de sus funciones de intérprete y acompañar a la usuaria a realizar otros trámites o bien la proveedora sugería que la usuaria podría llamarla en el caso de que necesitara su ayuda. Sorprendentemente, en ninguno de estos casos las intérpretes ponen por delante su perfil profesional especificando qué entra dentro del marco de sus funciones y qué no.

\section{Extracto 5. T=tutora; $\mathrm{I}=$ intérprete}

T: Sí. Y si hay que hablar con las familias, en este caso, como no voy a poder entenderme, tal vez te podría llamar a ti para que se lo expliques.

I: 下次老师要跟您的小孩子谈论, 对不对? 老师说如果说一下再来解释一下, 面谈, 老 师告诉你会下次是跟小孩儿谈论什么样子的情况, 如果您觉得听不懂的话, 我可以 $(\mathrm{xxx})$, 我可以帮你。

Luego la profesora hablará con su hijo, ¿verdad? Si una vez hayan hablado, hayan tenido la entrevista, ella quiere explicarle lo que han hablado con el niño, si usted no la entiende, yo puedo, puedo ayudar.

En un tercer punto rico, la tutora propone a la usuaria que sea su hijo quien le haga de intérprete en situaciones futuras. Sólo una de las intérpretes puntualiza y explica, a través de una adición de información, las desventajas que esto podría tener. Por su lado, cuatro de las intérpretes transmiten esta información sin ningún tipo de intervención por su parte. Observemos cómo, tras la reflexión que le hace la intérprete, la tutora se convence de que la idea de que el hijo haga de intérprete no es la mejor solución.

\section{Extracto 6. T=tutora; $\mathrm{I}=$ intérprete}

T: Y si no, pues el mismo hijo puede hacer de ... porque yo, normlamente, cuando hago estas entrevistas, llamo a los padres, no? Pero en su caso, que no podré tener este contacto, pues a través del hijo sí que le diré: "explícale esto a tu madre" y tal. Y si hubiera algún problema, pues ya te lo digo a ti.

I (dirigiéndose a la tutora): Sí, pero piensa que el hijo, muchas veces, o sea... yo he estado en situaciones de estas y los hijos no lo explican todo. Si os queréis mantener, o sea, transmitirle toda la información, es mejor que le hagas una reunión a parte.

T: Vale, tal vez sí que tienes razón. 
Por último, en otras dos ocasiones, las proveedoras realizan un comentario despectivo referido directamente a la usuaria. Con la excepción de dos únicos casos, el resto de intérpretes deciden suavizar el mensaje y no transmitir la dureza del mensaje original. Asimismo, algunas intérpretes deciden omitir directamente el mensaje. Se observa una tendencia generalizada a suavizar, neutralizar o incluso omitir comentarios discriminatorios o racistas por parte de los representantes de la administración hacia el usuario o su cultura. Según apuntaron las intérpretes en las entrevistas retrospectivas, el motivo de ello es siempre evitar el conflicto entre las partes. Ofrecemos a continuación un ejemplo ilustrativo de las estrategias que han aplicado las cinco intérpretes:

Extracto 7. T=tutora; $\mathrm{I} 1=$ intérprete $1 ; \mathrm{I} 2=$ =intérprete 2

T: ¿Por qué ha tardado tanto tiempo en venir a solicitar escuela? (dirigiéndose solo a la intérprete) Desde luego, habría podido venir antes...

\section{I1: [为什么还没找? 孩子为什么还没上学了? 为什么你没有早一点来了?}

¿Por qué no has buscado aún? Y ¿cómo es que el niño todavía no ha ido a la escuela? ¿Por qué no has venido antes?

I2: 你为什么没有早一点来到这里?

¿Por qué no has venido antes aquí?

I3: 她说你为什么那么长时间 没有过来? 你可以更早过 来, 为什么?

Dice que ¿por qué has tardado tanto a venir? Habrías podido venir antes, ¿por qué?

I4: 阿姨, 您小孩子已经来了两个月了, 不是吗? 现在才来问这个报学校上学=

Ayi, su hijo ya lleva aquí hace dos meses, ¿verdad? Y no ha venido hasta ahora a preguntar para matricularlo a la escuela

I5: (silencio). (Omite toda la idea)

En el caso de la primera intérprete, mediante el tono de voz y el tipo de preguntas (¿por qué no...?) se transmite parcialmente la crítica a la usuaria, aunque de una manera menos «amenazante» desde el punto de vista de la cortesía lingüística. La intérprete número 2 decide neutralizar el mensaje y eliminar el tono despectivo directamente. La tercera intérprete es la única que mantiene el tono duro del original $y$, además, en la entrevista a posteriori afirma que ella siempre intenta mantener el tono, aunque los comentarios que tenga que transmitir puedan ser despectivos. La cuarta intérprete consigue suavizar el tono en cierta medida. No obstante, mediante el recurso al adverbio chino 才, que indica que una acción se realiza más tarde de lo esperado, denota de manera implícita cierta crítica. La última intérprete reacciona con un silencio, tras el cual omite toda la idea.

\section{Discusión de los resultados}

Este estudio experimental permite establecer una primera categorización de algunos recursos estratégicos que emplean los intérpretes en los Servicios Públicos ante problemas de distinta índole. En relación con los problemas lingüísticos (léxicos, básicamente), la estrategia de mayor recurrencia es la omisión, que contrasta con el poco 
recurso que los intérpretes hacen a la pregunta cuando se trata de aspectos terminológicos. En lo que respecta a la omisión, no podemos distinguir si estas son conscientes o no, solo en una única ocasión hemos podido recoger la reflexión de la intérprete sobre una omisión consciente, con el fin de no abrumar a la usuaria con demasiada información, algo que también encontraba Napier en su estudio de 2004. Aunque exponíamos al comienzo del artículo que el objetivo que nos proponemos no es valorar la adecuación de las estrategias empleadas, en el caso de las omisiones se pone de manifiesto que en la mayoría de los casos se podrían calificar como errores de interpretación, ya que las intérpretes estarían vulnerando el principio ético de exactitud y fidelidad e incurriendo en el peligroso terreno de filtrar el mensaje.

Asimismo, las intérpretes recurren más a la formulación de preguntas cuando los problemas están relacionados con los nombres propios concretos o con siglas. En relación a las preguntas, sin embargo, cuando se trata de aspectos extralingüísticos, observamos cómo las intérpretes se muestran cómodas y dispuestas a interrumpir y a solicitar aclaraciones o repeticiones. Si bien estas preguntas sirven para esclarecer, desambiguar, o confirmar información confusa, suelen ralentizar la interacción. Asimismo, sin una estrategia de transparencia posterior, si no hay enseguida una explicación a la otra parte de qué es lo que se ha preguntado, propician el establecimiento de diálogos monolingües con alguno de los interlocutores primarios, en los que el tercer participante queda excluido y se vuelve a vulnerar un principio ético fundamental: el de la transparencia.

En general, se observa un amplio recurso por parte de las profesionales a lo que Baraldi y Gavioli (2012) llaman coordinación reflexiva (reflexive coordination), es decir, a la actividad metacomunicativa cuyo objetivo radica en solucionar problemas mediante la aclaración, la ampliación, las preguntas, etc. En este sentido, las estrategias que permiten la coordinación reflexiva coinciden, en gran parte, con las estrategias propias de la mediación y la comunicación intercultural, como serían las traducciones perifrásticas, las omisiones selectivas, las explicaciones culturales o la suavización de elementos que pueden suponer una amenaza para la reputación (Berk-Seligson 1988) de uno de los interlocutores. Estos recursos estratégicos acercan a los profesionales hacia un tipo de mediación activa, que tiene por objetivo la reducción de las diferencias y la promoción del entendimiento mutuo. Estas estrategias pueden contribuir en algunos casos a resolver posibles conflictos, al empoderamiento del usuario y a la creación de vínculos de familiaridad, pero no podemos olvidar, sin embargo, que mediación e interpretación son actividades diferenciadas pero que, como en los ejemplos que hemos visto, se pueden ver entrelazadas. Durante una entrevista interpretada, el intermediario puede necesitar dejar de interpretar para interceder brevemente si no hay más remedio, como en el caso de que se produzcan malentendidos, por ejemplo. Es esencial, sin embargo, tener presente que, durante una entrevista interpretada, el objetivo del intermediario debe ser permitir que las partes se comuniquen autónomamente a pesar de la barrera lingüística (y de las diferencias culturales).

Asimismo, llama la atención el escaso recurso que hacen las intérpretes a las técnicas propias de la interpretación, ya sea el uso de la toma de notas o la reordenación y estructuración de la información. Sin embargo, teniendo en cuenta que sólo 2 de las profesionales de la muestra afirmaron tener formación en ISP, no es de extrañar que, ante fragmentos largos de discurso, enumeraciones, nombres propios o cifras, las intérpretes no muestren el automatismo de anotar y recurran a estrategias 
que entorpecen la fluidez de la comunicación, tales como la tendencia a pedir una pausa al hablante y a la fragmentación de las ideas largas en varias intervenciones o al uso de preguntas para ir comprobando que la información les ha quedado clara. También destaca el poco uso de estrategias de tipo profesional específicas de la ISP, como el conocimiento y aplicación tanto de los principios básicos que recogen los principales códigos deontológicos de esta profesión (exactitud, confidencialidad, imparcialidad, transparencia, etc.), así como de los estándares profesionales o pautas de buenas prácticas más habituales.

\section{Conclusiones}

Las estrategias de interpretación, si se integran de manera regular y consciente en la formación, se consiguen automatizar con el paso del tiempo. Por esta razón, existe un acuerdo extendido entre los formadores de intérpretes en torno a la importancia que tiene formar a los futuros intérpretes sobre las necesidades estratégicas, tanto desde la teoría como desde la práctica.

Si las tareas de mediación e interpretación las realiza un mismo profesional como sucede en el caso español que se ve ilustrado en los ejemplos de este artículoes necesario que estos profesionales reciban formación específica tanto en técnicas de mediación estratégica breve y no intrusiva (Bancroft y Rubio-Fitzpatrick 2011; Bancroft et al. 2015), apropiadas para situaciones de entrevista en las que la autonomía comunicativa del proveedor del servicio y del usuario es un objetivo prioritario, como en técnicas de interpretación. De la misma manera, es imprescindible que los mismos profesionales reciban formación para que sean capaces de identificar de forma clara entre cuándo están interpretando y cuándo están mediando. Solo si pueden identificar cuál es su papel, podrán optar por aquellas decisiones estratégicas acordes con este.

Asimismo, la frecuente vulneración de los principios profesionales (exactitud, precisión, transparencia, etc.), la falta de estrategias que les permitan solucionar conflictos éticos y la multiplicidad de situaciones en las que el intérprete, como coordinador de la interacción, debe desempeñar una participación más activa (mediante preguntas, clarificaciones, etc.) ponen claramente de manifiesto la necesidad de un marco deontológico en España que pueda guiar, pautar y homogeneizar la intervención de los intérpretes. La falta de un código ético compartido deja a los intérpretes sin la brújula según la cual guiar sus decisiones y que les ofrezca un marco de principios y valores comunes y compartidos. Por esta razón, urge el desarrollo y difusión de un código ético profesional aplicable al contexto español y una mayor integración del contenido de los principios y estándares, recogidos en la bibliografía relevante, en la formación de intérpretes en España. Queda claro que para que usuarios y proveedores mantengan su autonomía (y no se vean desempoderados por la barrera lingüística y cultural), es necesario que durante la entrevista sepan en todo momento qué está pasando para poder tomar decisiones informadas en su proceso comunicativo. Para asegurar la transparencia, es preciso mantener siempre durante la entrevista el objetivo de transferir mensajes con precisión y fidelidad; transferir mensajes en estilo directo en vez de estilo indirecto y solo interceder cuando sea necesario y asegurándose de que todos los participantes sepan que se trata de una intervención de la intérprete y en qué ha consistido dicha intervención. 
En cuanto a las limitaciones del estudio, la más obvia es el carácter artificial de las situaciones analizadas. Aunque se intentó recrear al máximo las situaciones para que fueran lo más parecidas posibles a la realidad, los intérpretes tenían muy presente que estaban interpretando en una situación recreada, con lo cual esta sensación pudo haber influido en sus prestaciones. En este sentido, en la entrevista retrospectiva, la intérprete 1 admitía no haber sentido la presión por hacerlo bien que solía sentir en una situación real de trabajo, porque en todo momento sabía que estaba interpretando en una situación ficticia. Ante esta limitación evidente, en la actualidad hemos empezado un nuevo proyecto de investigación "TIPp: Traducción e Interpretación en los Procesos Penales", financiado por el Ministerio español de Economía y Competitividad, que se basa en el estudio del componente estratégico de los intérpretes en los servicios públicos del ámbito judicial y en el cual se está trabajando con un corpus de juicios reales de los tribunales de lo penal.

De la misma manera, habría sido útil realizar entrevistas retrospectivas tras un análisis detallado previo que hubiera permitido seleccionar los fragmentos concretos de discurso sobre los que se quería reflexionar con las intérpretes. Esto habría ayudado a determinar de forma más precisa, por ejemplo, la naturaleza de las omisiones. No obstante, por motivos de tiempo, las entrevistas retrospectivas tuvieron lugar poco después de las simulaciones y en ellas se vieron únicamente los problemas más evidentes que los investigadores habían podido identificar durante la simulación.

\section{NOTAS}

1. El grupo MIRAS está formado por un equipo de investigadores en ISP procedentes tanto del mundo académico como profesional. Para más información sobre el perfil de sus integrantes, véase: http:// grupsderecerca.uab.cat/miras/es/content/miembros.

2. "Problemas y estrategias de traducción e interpretación en el ámbito socioeducativo. Estudio de situaciones de interpretación de chino, rumano y árabe" [FFI2001-23905]. El objetivo general de este proyecto, desarrollado entre los años 2011 y 2014, fue estudiar el comportamiento estratégico del intérprete en los servicios públicos del ámbito socioeducativo ante problemas de interpretación de diversa índole.

3. NisKa, Helge (1998): Explorations in translational creativity: strategies for interpreting neologisms, no publicada. Workshop of the Institute for Interpretation and Translation Studies, University of Stockholm. Estocolmo, 8 de agosto de 1998. Consultado el 3 de septiembre de 2016, <http://lisa. tolk.su.se/kreeng2.htm>.

4. En esta página web se pueda encontrar más información sobre el proyecto TIPp: http://pagines. uab.cat/tipp/.

\section{REFERENCIAS BIBLIOGRÁFICAS}

Abuín, Marta (2007): El proceso de interpretación consecutiva. Un estudio del binomio problema/ estrategia. Granada: Editorial Comares.

Arumí, Marta (2012): Problems and Strategies in Consecutive Interpreting: A Pilot Study at Two Different Stages of Interpreter Training. Meta. 57(3):118-137.

Arumí, Marta y VArgas-URPí, Mireia (2014): Estrategias de interpretación en los servicios públicos en el ámbito educativo: estudio de caso en la combinación chino-catalán. Intralinea. 16:1-10.

Bancroft, Marjory y Rubio Fitzpatrick, Lourdes (2011): The Community Interpreter. Cross Cultural Communications. Columbia: Culture and Language Press.

Bancroft, Marjory, García-Beyaert, Sofía, Allen, Katherine, et al. (2015): The Community Interpreter. An International Textbook. Medical, Educational and Social Services Interpreting. Columbia: Culture and Language Press. 
Baraldi, Claudio y Gavioli, Laura, eds (2012): Coordinating Participation in Dialogue Interpreting. Amsterdam/Philadelphia: John Benjamins.

BARTLOMIEJCy K, Magdalena (2006): Strategies of simultaneous interpreting and directionality. Interpreting. 8(2):149-174.

Berg-Seligson, Susan (1988): The impact of politeness in witness testimony: The influence of the court interpreter. Multilingua. 7:411-439.

Denzin, Norman K. (1978): The research act: A theoretical introduction to sociological methods. New York: McGraw-Hill.

Englund Dimitrova, Birgitta (1997): Degree of interpreters' responsibility in the interaction process in community interpreting. In: Silvana E. CARr, Roda Roberts, Aideen Dufour y Dini Steyn, eds. The Critical Link: Interpreters in the Community. Amsterdam: John Benjamins, 147-164.

GAVIOLI, Laura y BARALDI, Claudio (2012): Interpreter-mediated interaction in healthcare and legal Settings. Interpreting. 13(2):205-233.

GiLE, Daniel (2002): Conference Interpreting as a Cognitive Management Problem. In: Franz Póchhacker y Miriam Shlesinger, eds. The Interpreting Studies Reader. London: Routledge, 162-176.

GiLe, Daniel (1995/2012): Basic Concepts and Models for Interpreter and Translator Training. Amsterdam/Philadelphia: John Benjamins.

Gumul, Ewa (2006): Explicitation in Simultaneous Interpreting. A Strategy or a By-product of Language Mediation? Across Languages and Cultures. 7(2):171-190

Hurtado, Amparo (2001): Traducción y traductología. Madrid: Cátedra.

Ivanova, Adelina (2000): The use of retrospection in research on simultaneous interpreting. In: Sonja TirkKonen-Condit y Riita Jä̈̈skeläInen, eds. Tapping and Mapping the Processes of Translation and Interpreting. Amsterdam/Philadelphia: John Benjamins, 27-52.

HönIG, Hans G. and Kussmaul, Paul (1982): Strategie der Übersetzung [Strategy of Translation]. Tübingen: G. Narr.

Kalina, Sylvia (1998): Strategische Prozesse beim Dolmetschen [Strategic Processes in Interpreting]. Tübingen: G. Narr.

Kalina, Sylvia (2000): Interpreting Competences as a Basis and a Goal for Teaching. The Interpreters' Newsletter. 10:3-32.

Kalina, Sylvia (2015): Strategies. In: Franz Pöchнacker, ed. Routledge Encyclopedia of Interpreting Studies. London/New York: Routledge.

Kohn, Kurt y Kalina, Sylvia (1996). The Strategic Dimension of Interpreting. Meta. 41(1):118-138.

LI, Xiandong (2003): Are Interpreting Strategies Teachable? Correlating Trainees' Strategy Use with Trainers' Training in the Consecutive Interpreting Classroom. The Interpreter's Newsletter. 18:105-128.

Mason, Ian y Stewart, Miranda (2001): Interactional pragmatics, face and the dialogue interpreter. In: Ian MAson, ed. Triadic Exchanges: Studies in Dialogue Interpreting. Manchester: St. Jerome, 51-70.

MERLINI, Raffaela y FaVARon, Roberta (2003): Community interpreting: re-conciliation through power management. The Interpreters' Newsletter. 12:205-229.

Moser-Mercer, Barbara, Frauenfelder, Ulrich, Casado, Beatriz y Künzli, Alexander (2000): Searching to define expertise in interpreting. In: Kenneth Hyltenstam y Birgitta Englund Dimitrova, eds. Language processing and interpreting. Amsterdam: John Benjamins, 1-21.

NAPIER, Jemina (2004): Interpreting omissions. A new perspective. Interpreting. 6(2):117-142.

Niska, Helge (2002): Community Interpreter Training. In: Giuliana Garzone y Maurizio Viezzi, eds. Interpreting in the $21^{\text {st }}$ century. Challenges and opportunities. Philadelphia: John Benjamins, 135-146.

NorD, Christiane (1988): Textanalyse und Übersetzen [Text Analysis and Translation]. Heidelberg: J. Groos Verlag.

PACTE (2011): Results of the Validation of the PACTE Translation Competence Model: Translation Problems and Translation Competence. In: Cecilia Alvstad, Adelina Hild y Elisa- 
bet Tiselius, eds. Methods and Strategies of Process Research: Integrative Approaches in

Translation Studies. Amsterdam: John Benjamins, 317-343.

PöchHACKer, Franz (2004): Introducing Interpreting Studies. London/New York: Routledge.

PöchHACKer, Franz (2010): The role of research in interpreter education. Translation \& Interpreting. 2(1):1-10

PöchнаскеR, Franz, ed. (2015): Routledge Encyclopedia of Interpreting Studies. London/New York: Routledge.

Pöllabauer, Sonja (2004): Interpreting in asylum hearings: issues of role, responsability and power. Interpreting. 6(2):143-180.

RiCCARDI, Alessandra (1996): Language-specific strategies. In: Cay Dollerup y Viveke APPEL, eds. New Horizons Teaching Translation and Interpreting. Amsterdam/Philadelphia: John Benjamins, 213-221.

RicCARdi, Alessandra (1998): Interpreting Strategies and Creativity. In: Ann BeylaARd-OzerofF, Jana KrÁvolvá y Barbara Moser-Mercer, eds. Translators' Strategies and Creativity. Amsterdam/Philadelphia: John Benjamins, 171-179.

RiCCARDI, Alessandra (1999): Interpretazione simultanea: strategie generali e specifiche. In: Caterina Falbo, Mariachiara Russo y Francesco Straniero, eds. Interpretazione simultanea e consecutiva: Problemi teorici e metodologie didattiche [Simultaneous and consecutive interpretation: Theoretical problems and teaching methods]. Milan: Ulrico Hoepli, 161-174.

Roy, Cynthia (2000): Interpreting as a Discourse Process. New York/Oxford: Oxford University Press.

Sales Salvador, Dora (2003): Interacción comunicativa intercultural con inmigrantes procedentes de la cultura china. In: Grup CRIT, ed. Claves para la comunicación intercultural. Análisis de interacciones comunicativas con inmigrantes. Castellón de la Plana: Universitat Jaume I, 123-148.

Sanchez Perez, Marta (2009): La mediación intercultural en el ámbito sanitario. El caso de los pacientes de origen chino. Tesina de maestría, no publicada. Castellón de la Plana: Universitat Jaume I.

Sanchez Perez, Marta (2015): Mediación interlingüistica e intercultural en el ámbito de la salud sexual y reproductiva: estudio de casos con usuarias de origen chino. Tesis de doctorado, no pulicada. Castellón de la Plana: Universitat Jaume I.

SunNari, Marianna (1995): Processing Strategies in Simultaneous Interpretating: Saying it All vs. Synthesis. In: Jorma Tommola, ed. Topics in Interpreting Research. Turku: University of Turku Centre for Translation and Intepreting, 109-119.

VAlero-Garcés, Carmen (2005): Terminology and Ad Hoc Interpreters in Public Services. An Empirical Study. JosTrans. 3:75-96.

VARGAS-URPÍ, Mireia (2012): La interpretació als serveis públics i la mediació intercultural amb el collectiu xinès a Catalunya [Public service interpretation and intercultural mediation with the Chinese community in Catalonia]. Tesis de doctorado, no pulicada. Barcelona: Universitat Autònoma de Barcelona.

VARGAS-Ur Pí, Mireia (2015): Problems and strategies in public service interpreting as perceived by a sample of Chinese-Catalan/Spanish interpreters. Perspectives: Studies in Translatology. 22:666- 678 .

Vik-Tuovinen, Gun-Viol (2002): Retrospection as a method of studying the process of simultaneous interpreting. In: Giuliana Garzone y Maurizio VIEzzI, eds. Interpreting in the 21st Century. Amsterdam: John Benjamins, 63-71.

WADENSJÖ, Cecilia (1998): Interpreting as Interaction. London: Longman.

WADENSJÖ, Cecilia (2001): Approaching interpreting through discourse analysis. In: Daniel GILE, Helle Vrønning Dam, Friedel Dubslaff, Bodil Martinsen y Anne Schjoldager eds. Getting Started in Interpreting Research. Amsterdam: John Benjamins, 185-198.

WANG, Binhua (2012): Interpreting Strategies in Real-life Interpreting. Corpus-based Description of Seven Professional Interpreters' Performance. Translation Journal. 16(2). Consultado el 15 de septiembre de 2016, <http://translationjournal.net/journal/60interpreting.htm>. 